\title{
Analyzing the use of linking words in concept maps designed for pathophysiology learning in medicine
}

\author{
Marta Fonseca $^{1,2}$, Beatriz Oliveira ${ }^{1}$, Inês Canha ${ }^{1}$, Hélder Dores ${ }^{1}$, Madalena Pinheiro \\ Santos $^{1}$, Vasco Cremon Lemos ${ }^{1}$, Alexandra Verdasca ${ }^{1}$, Mariana Branco ${ }^{1}$, Ana Rita \\ Póvoa $^{1}$, Pedro Carreiro-Martins ${ }^{1,2}$, António Rendas ${ }^{1}$, Nuno Neuparth ${ }^{1,2}$ \\ ${ }^{1}$ Pathophysiology Department, NOVA Medical School, Lisbon, Portugal, ${ }^{2}$ Comprehensive \\ Health Research Center (CHRC), NOVA Medical School, Lisbon, Portugal.
}

\begin{abstract}
Pathophysiology at NOVA Medical School adopted the concept mapping methodology to promote the visual display of pathophysiological reasoning and learning, based on clinical vignettes. The objective of this project is to identify and label the linking words, in order to study their role in the structure and organization of the concept maps constructed by the students. We used an adopted classification of linking words, categorized in 5 groups: dynamic, static, illustrative, definition and clinical case information. At the end of the semester the concept maps related to the respiratory (mid-semester) and endocrine systems (end of semester) were analyzed and compared. We found linking words not included in any of the five categories, thus a group named "other" has been created. Statistically significant differences were found in dynamic and "other" categories ( $p=0,049$ and $p=0,011$, respectively; Wilcoxon signed-rank test). The dynamic words were the most commonly used, probably reflecting students' need to better describe pathophysiological mechanisms, and the difference found was probably due an improvement in the learning process and concept maps building technic. It would be interesting next year to conduct a more detailed analysis, increasing the sample and ensuring a more robust dataset.
\end{abstract}

Keywords: Concept maps; linking words; critical thinking; medical education; pathophysiology. 


\section{Introduction}

Pathophysiology teaching at NOVA Medical School (NMS) adopted the concept mapping methodology in 2002 (Rendas et al, 2006), to be used during the tutorial sessions, in order to promote the visual display of pathophysiological reasoning and learning, based on clinical vignettes. A concept map (CM) consists of nodes (conceptual words/phrases), connected by linking lines which are unidirectional arrows, with a linking word/phrase describing the semantic relationship between the nodes (Novak \& Cañas, 2008). The use of linking words is one of the most important features of creating a concept map, since whatever linking words the student uses will change the relationship between the concepts and therefore the meaning portrayed on the map (Daley et al, 2016). In our case, the purpose is that these semantic relationships aim to reflect the understanding of mechanisms of disease, the pathophysiology, and their scientific fundamentals.

The objective of this project is to identify, and label, the linking words/phrases in order to study their role in the structure and organization of the CM. This approach was used for the first time in our curricula and started with developing a classification of the words/phrases in five groups, as will be described below, and analyzing their distribution and coherence in the maps.

This preliminary approach is part of our larger project aiming to develop a score methodology to assess concept maps in medical education, presented last year at HEAd'20 (Fonseca et al, 2020), wich did not address specifically the role of the linking words. In this context, we considered the use of appropriate linking words as an important scaffold to the display of the learning process and this facet was not considered in our initial classification. To reach this goal we opted to standardize the linking words and adopted the classification proposed by O'Donnell (O'Donnell et al, 2002), based on the main categories used by McCagg and Dansereau (McCagg \& Dansereau, 1991). Furthermore, we added three new groups because of the context of medical education using clinical vignettes: illustrative/examples, definitions and clinical case information. This approach reflects our progressive awareness of the importance of using standardized steps inserted in the original methodology of concept mapping as proposed by Novak (Novak \& Cañas, 2008) and Daley (Daley et al, 2016).

The purpose of this project is to analyze how linking word/phrases are applied during the construction of concept maps in the specific context of pathophysiology teaching and learning at NMS. 


\section{Material and Methods}

\subsection{General Information}

The pathophysiology tutorial sessions are based on an organ/body system approach, covering five systems: digestion, respiration, circulation, renal and body fluids and endocrine (Fonseca et al, 2020), and lasts one semester of the second year of the curriculum. For each system, students received a clinical vignette (patient case) and eight mini-maps were constructed based on eight core concepts leading to the creation of a final CM (Figure 1). This report only addresses the analysis of two of the final CMs.

In the 2020-2021 academic year, the class was composed of 233 newly enrolled students, divided into 18 tutorial groups. Each tutorial group was attributed to one of the 14 pathophysiology tutors.
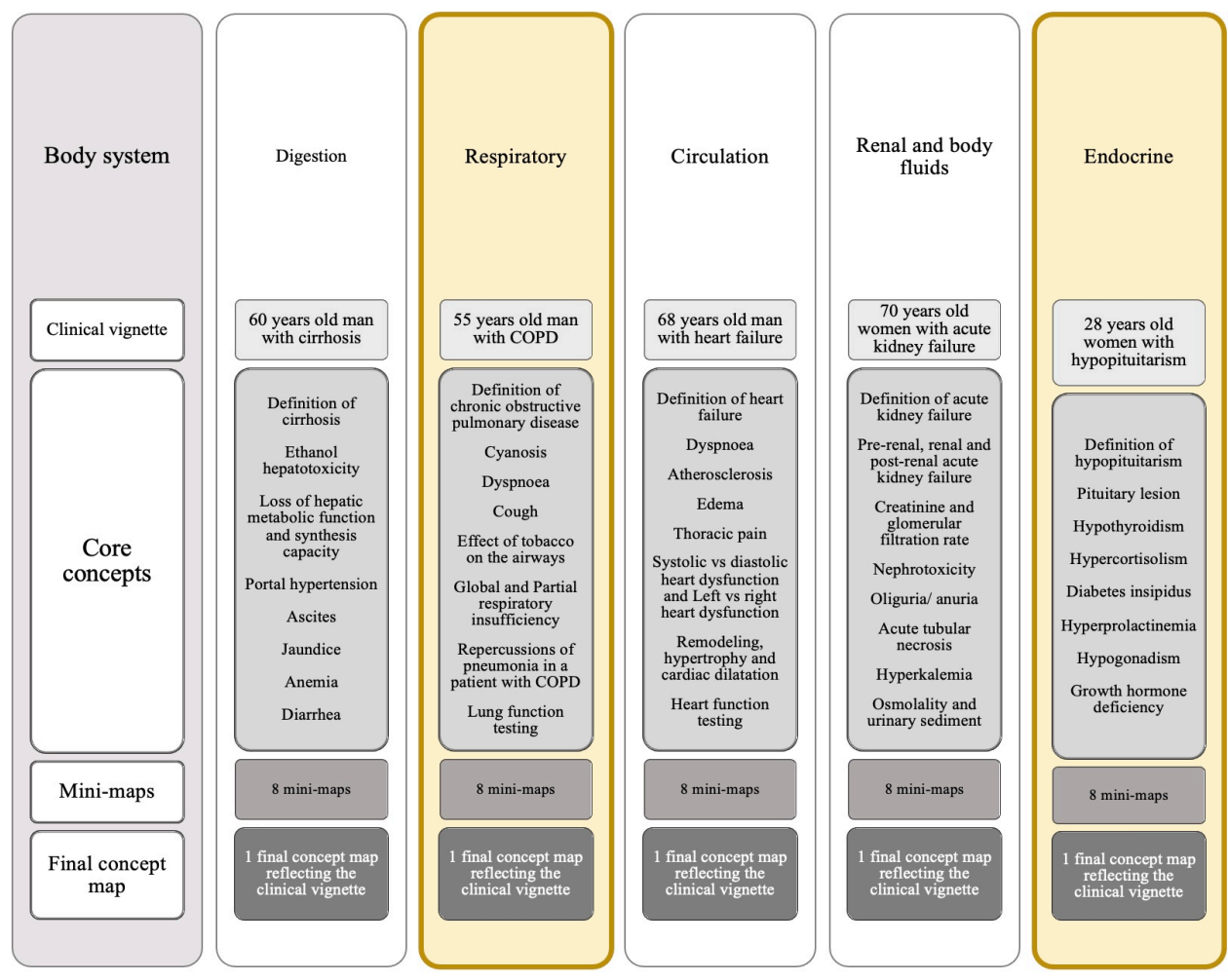

Figure 1. Organization of the pathophysiology tutorial sessions based on an organ/body system approach. For each system, students were asked to construct eight mini-maps, based on the identified core concepts, and a final concept map reflecting the clinical vignette. In this project we considered the respiratory and endocrine final concept maps. Source: the authors. 


\subsection{Concept maps' linking words in the 2020-2021 academic year}

In the beginning of the current academic year, students were instructed to use a standardized group of linking words in the construction of their concept maps as stated in the introduction.

Our classification of the linking words (Table 1) was adopted from the work of O'Donnell (O’Donnell et al, 2002) as:

(i) dynamic: a link word/phrase, that represents a causal, a temporal, a quantified changing relation between nodes;

(ii) static, a link word/phrase, that represents a complementary or supplementary or clarifying relation between nodes with no direct cause-effect;

(iii) illustrative, a link word/phrase, that represents a single label connecting nodes;

(iv) definition, a link word/phrase, represents a consensual label, based on scientific knowledge connecting nodes;

(v) clinical case information, a word/phrase, that represents the information, displayed in the map which is provided in the clinical vignette.

Table 1. Classification and list of concept maps' linking words within each group.

\begin{tabular}{|c|c|c|c|c|c|}
\hline \multicolumn{6}{|c|}{ Linking Words } \\
\hline \multicolumn{2}{|c|}{ Dynamic } & Static & Illustrative & Definitions & $\begin{array}{c}\text { Vignette } \\
\text { information }\end{array}$ \\
\hline $\begin{array}{l}\text { by means of } \\
\text { causes } \\
\text { compromises } \\
\text { constrains } \\
\text { culminates } \\
\text { determines } \\
\text { favors } \\
\text { follow } \\
\text { infuences }\end{array}$ & $\begin{array}{l}\text { unhibits } \\
\text { leads to } \\
\text { promotes } \\
\text { results in } \\
\text { sequences } \\
\text { stimulates } \\
\text { though } \\
\text { triggers }\end{array}$ & $\begin{array}{c}\text { typifies } \\
\text { includes } \\
\text { characterizes } \\
\text { charecterized } \\
\text { by } \\
\text { manifests } \\
\text { itself } \\
\text { present }\end{array}$ & $\begin{array}{c}\text { analogies } \\
\text { namely } \\
\text { example } \\
\text { dor example }\end{array}$ & is defined as & $\begin{array}{c}\text { in the clinical } \\
\text { case }\end{array}$ \\
\hline
\end{tabular}

At the end of the curricular unit, junior students from the third year of NMS, who had acted as mentors to assist the pathophysiology students in the construction of the maps, analyzed the final respiratory (mid-semester) and endocrine system CM (end of semester), by quantifying the linking words based on the above-mentioned classification. Their analysis was supervised by a senior student (final year), with a significant experience in scoring concept maps.

The prerequisites were as follows: only final CMs were analyzed, and students, juniors and senior, did not analyze the CMs they helped to construct. 


\subsection{Statistical analysis}

An exploratory analysis of the linking words was carried out. The Wilcoxon signed-rank test was used to compare the number of linking words between final respiratory and endocrine system CMs constructed during the tutorial sessions, in each class. The level of significance considered was $\alpha=0.05$. Data analysis was performed using STATA (StataCorp LP, Stata Statistical Software: Release 15; Texas, U.S.A.).

\subsection{Pedagogical Council}

These activities were approved by the Pedagogical Council of NMS, which is the board legally responsible for regulating all of the academic procedures. It also includes representatives of medical students.

\section{Results}

From the eighteen classes in the course, seventeen final CMs per body system were analyzed (one CM from a tutorial group was missed). Of these seventeen CMs per system, eight final respiratory and eight final endocrine systems CMs, belonging to the same tutorial groups, were analyzed. Table 2 presents the number of linking words found on each of the CMs, grouped into the five pre-specified categories (Table 1). In some CMs we found linking words that could not be included in any of the 5 categories, thus a group "other" has been created which should always be lower than the sum of the words from the five categories. Statistically significant differences were found in dynamic words and other categories ( $p=0,049$ and $p=0,011$, respectively; Wilcoxon signed-rank test).

The most commonly used words in both respiratory and endocrine system's CMs were dynamic words, with a median number of $60.5(47.5 ; 79)$ and $77(60 ; 94)$ words per CM, respectively. These were followed by static words (median $16(12.5 ; 24.5)$ and $20(10 ; 45)$ ), illustrative words (median $6(2 ; 8.5)$ and $4(2.5 ; 12))$ and definitions (median $2.5(0.5 ; 6)$ and $2.5(1 ; 6.5))$.

\section{Discussion and Conclusions}

This work reflects the first analysis of our group performed on the CM's linking words. At the beginning of the semester, instructions were given to tutors and students with the list of linking words to use, as detailed in the body of this article. We found that, despite this preparation, a significant number of different words were used in all the CMs analyzed. This finding points to the need to further improve the initial list, reduce the heterogeneity of results between the tutors, and also to the potential benefit of dedicating more time in addressing the use of right terminology during the tutorial classes in the future academic years. This other category encompassed all the words that were not included in the pre-specified categories. It 
is noteworthy that its use decreased from the respiratory to the endocrine system, which may reflect the impact of students' learning process translated in to a more careful and appropriate use of these words in the CMs.

Table 2. Number of linking words in the respiratory and endocrine final concept maps (per category).

\begin{tabular}{|c|c|c|c|c|c|c|c|c|c|}
\hline & 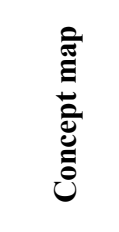 & 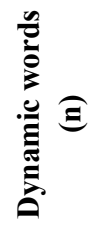 & 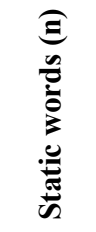 & 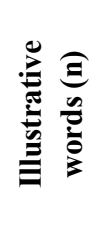 & 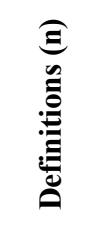 & 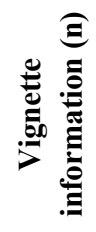 & $\begin{array}{c}\widehat{\Xi} \\
\widehat{\sigma} \\
\hat{\theta}\end{array}$ & 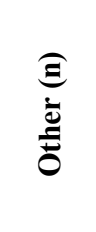 & 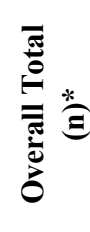 \\
\hline \multirow{9}{*}{ 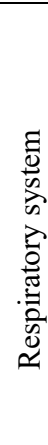 } & 1 & 46 & 27 & 0 & 0 & 0 & 73 & 11 & 84 \\
\hline & 2 & 58 & 13 & 0 & 1 & 36 & 108 & 37 & 145 \\
\hline & 3 & 49 & 12 & 6 & 16 & 30 & 113 & 60 & 173 \\
\hline & 4 & 44 & 22 & 10 & 5 & 16 & 97 & 12 & 109 \\
\hline & 5 & 84 & 14 & 4 & 3 & 0 & 105 & 80 & 185 \\
\hline & 6 & 63 & 9 & 6 & 7 & 18 & 103 & 37 & 140 \\
\hline & 7 & 74 & 42 & 12 & 0 & 0 & 128 & 22 & 150 \\
\hline & 8 & 89 & 18 & 7 & 2 & 0 & 116 & 81 & 197 \\
\hline & Total & 507 & 157 & 45 & 34 & 100 & 843 & 340 & 1183 \\
\hline \multirow{10}{*}{ 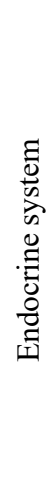 } & 1 & 78 & 44 & 2 & 8 & 12 & 144 & 2 & 146 \\
\hline & 2 & 52 & 11 & 1 & 1 & 3 & 68 & 32 & 100 \\
\hline & 3 & 98 & 20 & 13 & 15 & 18 & 164 & 34 & 198 \\
\hline & 4 & 54 & 20 & 18 & 5 & 35 & 132 & 7 & 139 \\
\hline & 5 & 155 & 46 & 11 & 2 & 0 & 214 & 73 & 287 \\
\hline & 6 & 66 & 9 & 5 & 1 & 26 & 107 & 17 & 124 \\
\hline & 7 & 76 & 47 & 3 & 1 & 0 & 127 & 15 & 142 \\
\hline & 8 & 90 & 7 & 3 & 3 & 12 & 115 & 74 & 189 \\
\hline & Total & 669 & 204 & 56 & 36 & 106 & 1071 & 254 & 1325 \\
\hline & $\mathrm{p}$ value $^{1}$ & 0.049 & 0.361 & 0.528 & 0.943 & 0.618 & 0.049 & 0.011 & 0.652 \\
\hline
\end{tabular}

* corresponds to the total sum of the dynamic, static, illustrative, definitions, vignette information and other linking words.

The selection of the eight final CMs per body system was made to avoid sample heterogeneity since, in the non-selected maps, the number of words in the other category exceeded the sum of the words used across the 5 pre-specified categories. This, once again, may reflect the need for further training of tutors to reduce their own heterogenous use of linking words, and for 
increased attention to the correct use of pre-specified categories' words at the beginning of the curricular unit and during the whole semester.

Although the sample used was small, it was interesting to see that most of the words used are dynamic, and that their use has numerically increased $(\mathrm{p}=0.049)$ from the respiratory map to the endocrine map ( $43 \%$ of the total overall in the respiratory and $51 \%$ in the endocrine). This fact may reflect students' need to use more of these words to better describe pathophysiological mechanisms, improving over the course of the semester due to the learning process for content and CM's building technic. The fact that these CMs are based on different pathologies (COPD and hypopituitarism, Figure 1), may also contribute for this difference. A numerical difference in the number of linking words used in both pathologies' $\mathrm{CMs}$ has been observed, althout it was not statistically significant (respiratory total $=1183$, endocrine total $=1325 ; \mathrm{p}=0.652$ ).

The difference found in the group other was statistically significant $(p=0.011)$, with a decrease in the use of these linking words from the respiratory to the endocrine system from $29 \%$ to $19 \%$, respectively. This data may reflect more precision in its use, and a preference to use words from the pre-specified categories. Nevertheless, no detailed analysis was performed regarding the labelling of these other words according to our proposed classification. The linking words included in statics, illustrations, definitions and vignette categories were the least used and there were no significant differences in their use. Although less used, these words still were considered as being a structural part of the CM. Although there seems to be some preference in the use of dynamic words, all word's categories must be present in an CM. Their number and proportion still need to be further studied and evaluated in order to also be used as a potential parameter for a future scoring of CMs, a topic that our group has been actively working on.

It would be interesting next year, following more investment in training of tutors and students regarding the use of linking words, to conduct a more detailed analysis of all the CMs constructed by the students, increasing the sample and ensuring a more robust dataset.

\section{Acknowledgements}

Grateful thanks are due to the teaching staff of pathophysiology, tutors Manuel Almeida, Patrícia Santos, Diana Ferreira, Miguel Proença, Ana Rita Franco, Luísa Quaresma, Vasco Gaspar and António Mesquita, and the junior students Sofia Ribeiro, Pedro Vilão Silva, Joana Vigeant Gomes and Margarida Flores that acted as mentors during the tutorial sessions. 


\section{References}

Daley B. J., Durning, S. J., Torre, D. M. (2016). Using Concept Maps to Create Meaningful Learning in Medical Education. MedEdPublish, 5(1), 1-29. https://doi.org/10.15694/mep.2016.000019.

Fonseca M., Oliveira B., Carreiro-Martins P., Neuparth N., Rendas A. (2020). Revisiting the role of concept mapping in teaching and learning pathophysiology for medical students. Adv Physiol Educ, 44(3):475-81. doi:10.1152/advan.00020.2020.

Fonseca M., Oliveira B., Martins P., Neuparth N., Rendas A.B. (2020). A score methodology to assess concept maps in medical education in the context of pathophysiology teaching. 6th International Conference on Higher Education Advances (HEAd'20), 867-74. doi: http://dx.doi.org/10.4995/HEAd20.2020.11163.

McCagg E. C., Dansereau D. F. (1991). A Convergent Paradigm for Examining Knowledge Mapping as a Learning Strategy. The Journal of Educational Research, vol. 84(6), 317 324.

Novak J. D., Cañas A. J. (2008) The Theory Underlying Concept Maps and How to Construct and Use Them. IHMC C,1-36. doi:Technical Report IHMC CmapTools 2006-01 Rev 2008-01.

O'Donnell A. M., Dansereau D. F., Hall R. H. (2002). Knowledge maps as scaffolds for cognitive processing. Educ Psychol Rev, 14(1), 71-86. doi: 10.1023/A:1013132527007.

Rendas A. B., Fonseca M., Pinto P. R. (2006). Toward meaningful learning in undergraduate medical education using concept maps in a PBL pathophysiology course. Adv Physiol Educ, 30(23), 23-29. doi:10.1152/advan.00036.2005. 